\title{
Silencing of MEG3 attenuated the role of lipopolysaccharides by modulating the miR-93-5p/PTEN pathway in Leydig cells
}

\author{
Xu Zhou', Jingliang He ${ }^{1}$, Jinbo Chen ${ }^{2}, Y u \mathrm{Cui}^{2}$, Zhenyu $\mathrm{Ou}^{2}$, Xiongbing $\mathrm{Zu}^{2^{*}}$ and Nenghui $\mathrm{Liu}^{1 *}$
}

\begin{abstract}
Background: Leydig cells reflect the activation of inflammation, decrease of androgen production, inhibition of cell growth and promotion of cell apoptosis under orchitis. Maternally expressed gene 3 (MEG3) exerts a crucial role in various human diseases, but under orchitis, the role and underlying molecular mechanism of MEG3 in Leydig cells remain unclear.

Methods: Lipofectamine 2000 was used for the cell transfections. qPCR and western blots assay were applied to assess the gene expression. ELISA assay was used to measure the TNFa, IL6 and testosterone secretion. CCK8 and EdU assay was employ to test the cell viability and proliferation respectively. Luciferase reporter and RIP assay were introduced to detect the binding of miR-93-5p with MEG3 and PTEN.

Results: Lipopolysaccharides (LPS) induced TNFa and IL6 secretion, lowered testosterone production, inhibited cell viability and proliferation, and induced cell apoptosis in Leydig cells. MEG3 was upregulated in Leydig cells treated with LPS and that knockdown of MEG3 inhibited the role of LPS in Leydig cells. MEG3 absorbed miR-93-5p and that suppression of miR-93-5p restored the role of silenced MEG3 in Leydig cells under LPS treatment. miR-93-5p inhibited PTEN expression and that over-expressed PTEN alleviated the effect of miR-93-5p in Leydig cells treated with LPS. LPS activated the MEG3/miR-93-5p/PTEN signalling pathway in Leydig cells.

Conclusions: This study revealed that MEG3 serves as a molecular sponge to absorb miR-93-5p, thus leading to elevation of PTEN expression in Leydig cells under LPS treatment, offering a theoretical basis on which to establish potential new treatment strategies for orchitis.
\end{abstract}

Keywords: Orchitis, Leydig cell, LPS, MEG3, ceRNA

\section{Introduction}

As a dominant cause of infertility, orchitis is defined as inflammation of one or both testicles [1]. The causes of orchitis include infections, trauma, and tumours, and the precise incidence of orchitis is also well known [2]. As male-specific cells, testicular Leydig cells perform the main function of synthesis and secretion of male

\footnotetext{
*Correspondence: csuzuxb@163.com; 327412826@qq.com

${ }^{2}$ Department of Urology, Xiangya Hospital, Central South University, 87 Xiangya Road, Changsha 410008, Hunan, China

${ }^{1}$ Reproductive Medicine Center, Xiangya Hospital, Central South University, 87 Xiangya Road, Changsha 410008, Hunan, China
}

hormones, including progesterone and testosterone, which promote spermatogenesis and male reproductive organ development and maintain secondary sexual characteristics and sexual function [3]. As an immuneinducing factor, bacterial lipopolysaccharides (LPS) can induce cell inflammation, inhibit cell proliferation and induce cell apoptosis [4]. LPS induces lipid peroxidation and apoptosis, decreases secretion of testosterone, disturbs spermatogenesis production and disrupts spermatogenesis in the testes $[5,6]$. Therefore, understanding the role and the underlying mechanism of LPS in growth

(C) The Author(s). 2021 Open Access This article is licensed under a Creative Commons Attribution 4.0 International License, which permits use, sharing, adaptation, distribution and reproduction in any medium or format, as long as you give appropriate credit to the original author(s) and the source, provide a link to the Creative Commons licence, and indicate if changes were made. The images or other third party material in this article are included in the article's Creative Commons licence, unless indicated otherwise in a credit line to the material. If material is not included in the article's Creative Commons licence and your intended use is not permitted by statutory regulation or exceeds the permitted use, you will need to obtain permission directly from the copyright holder. To view a copy of this licence, visit http://creativecommons.org/licenses/by/4.0/ The Creative Commons Public Domain Dedication waiver (http://creativecommons.org/publicdomain/zero/1.0/) applies to the data made available in this article, unless otherwise stated in a credit line to the data. 
and testosterone production in Leydig cells might aid in development of innovative strategies for the treatment of orchitis.

Increasing evidence shows that less than $2 \%$ of the active genome is transcribed to protein-coding genes, indicating that most transcripts are non-coding RNAs (ncRNAs) [7]. NcRNAs were roughly classified into small ncRNAs with a length of less than 200 nucleotides and long ncRNAs (lncRNAs) with a length greater than 200 nucleotides $[8,9]$. Although once considered "transcriptional noise", lncRNAs play an important role in a variety of cellular progresses, including chromatin remodelling, cell proliferation and cycling, cell death, metastasis, development and tumourigenesis via regulation of genes at the transcriptional, post-transcriptional and translational levels [10-13].

LncRNAs exert influence in a great variety of human disease, including tumours and cancer, nervous systemrelated diseases, urogenital-related system diseases, cardiovascular-related diseases, gastrointestinal systemrelated diseases, embryonic development-related diseases and immune system-related diseases [14-16]. Sofia Boeg Winge and colleagues screened differential expressed lncRNAs in fixed paraffin-embedded testicular tissue samples and displayed the disturbance during differentiation of Leydig and Sertoli cells [17]. However, no literature is available to further elucidate the role and underlying mechanism of lncRNAs in the Sertoli cells of orchitis.

Located in chromosome 14q32, IncRNA maternally expressed gene 3 (MEG3) acts as a tumour suppressor and is involved in physiological and pathological progression of various human diseases [14, 18]. MEG3 has been found to play a crucial role in various inflammation-related diseases. Zhaolin Wang and colleagues reported that knockdown of MEG3 attenuated LPS-induced inflammatory injury in ATDC5 cells [19]. However, there is a lack of focus in the literature on the functional roles and underlying mechanism of MEG3 in Leydig cells under orchitis.

Therefore, this study aimed to investigate the role and further dissect the underlying molecular mechanism of MEG3 in Leydig cells under orchitis. This study successfully elucidated the role and underlying mechanism of MEG3 in Leydig cells under LPS and revealed a novel regulatory signalling pathway that offers a certain degree of potential for orchitis treatment.

\section{Materials and methods}

\section{Cell culture, treatment and transfection}

Human Leydig cells were purchased from ScienCell Research Laboratories (ScienCell, San Diego, California, USA) and routinely conserved in our labs and cultured in Dulbecco's modified Eagle medium (DMEM; GIBCO,
NY, USA) supplemented with $10 \%$ foetal bovine serum (FBS; Invitrogen, CA, USA), $10 \mathrm{U} / \mathrm{mL}$ penicillin and $10 \mu \mathrm{g} / \mathrm{mL}$ streptomycin. Cells were maintained in a $37^{\circ} \mathrm{C} / 5 \% \mathrm{CO}_{2}$ humidified incubator. LPS (Sigma-Aldrich, MO, USA) was dissolved in the medium. The cells were treated with LPS (Sigma-Aldrich, MO, USA) at different concentrations for approximately $48 \mathrm{~h}$. Transfection of small interfering RNA (siRNAs), miR-93 inhibitor, miR-93 mimics and control small RNAs (GenePharma, Shanghai, China) was performed using Lipofectamine 2000 (Thermo Fisher Scientific, CN, USA) over a period of approximately $48 \mathrm{~h}$.

\section{RNA isolation and real-time quantitative PCR}

Trizol reagent (Junxin, Suzhou, China) was introduced to extract the total RNA. A NanoDrop 2000 (Thermo Fisher) instrument was used to measure the concentration and purity of total RNA. A reverse transcription kit (Takara, Dalian, China) was applied to synthesize the first-strand cDNA. The $2 \times$ SYBR Green qPCR Mix (Junxin, Suzhou, China) was selected to perform qPCR analysis. The expression levels for lncRNA and mRNA were normalized to $\beta$-actin expression, and the expression levels for miRNAs were relative to the U6 expression. The $2^{-\Delta \Delta C t}$ method and arbitrary units were introduced to calculate the relative fold change. The primers used in this study are listed in Table 1.

\section{Western blot analysis}

The radio immunoprecipitation assay (RIPA) (Takara, Dalian, China) was introduced to isolate the protein. The BCA protein detection kit (Junxin, Suzhou, China) was applied to measure the concentration of protein. The proteins ( $30 \mu \mathrm{g}$ in each lane) were separated via sodium dodecyl sulfate-polyacrylamide gel electrophoresis (SDS-PAGE) and transferred onto polyvinylidene fluoride (PVDF) membranes (Millipore, USA). The membranes were blocked in $5 \%$ fat-free milk and exposed to different primary antibodies at $4{ }^{\circ} \mathrm{C}$ overnight. The membranes were incubated with horseradish peroxidase-conjugated IgG. The immunoreactive protein bands were detected with a chemiluminescence (ECL) reagent (Junxin, Suzhou, China) and the Bio-Rad ChemiDocTM XRS system. $\beta$-Actin was measured as a loading control. The antibodies used in this study are listed below: The anti-PTEN antibody (Ab267787, Abcam, Cambridge, British), anti-Bcl-2 antibody (Ab32124, Abcam, Cambridge, British), anti-Bax antibody (Ab182734, Abcam, Cambridge, British), anti- $\beta$-Actin (Ab207604, Abcam, Cambridge, British) and Goat Anti-Rabbit IgG H\&L (HRP) (Ab 6721, Ab207604, Abcam, Cambridge, British) were used according to the protocol. 
Table 1 Primers used in this study

\begin{tabular}{|c|c|c|}
\hline Gene & primer & Sequence \\
\hline \multirow[t]{2}{*}{ MEG3 } & Forward & 5'- GGCAGGATCTGGCATAGAGG - 3' \\
\hline & Reverse & 5'- CGAGTCAGGAAGCAGTGGGTT-3' \\
\hline \multirow[t]{2}{*}{ PTEN } & Forward & 5'- ACCCACACGACGGGAAGACA - 3' \\
\hline & Reverse & 5'- CTGTTTGTGGAAGAACTCTACTTTGATATCAC - 3' \\
\hline \multirow[t]{2}{*}{$\beta$-actin } & Forward & 5'- CATTCCAAATATGAGATGCGTTGT - 3' \\
\hline & Reverse & 5'- TGTGGACTTGGGAGAGGACT - 3' \\
\hline \multirow[t]{2}{*}{$\operatorname{miR}-93-5 p$} & Forward & 5'- GTCACAAAGUGCUGUUCGUGC-3' \\
\hline & Reverse Transcription & 5'-GTCGTATCCAGTGCAGGGTCCGAGGTATTCGCACTGGATACGACCTACCTG - 3' \\
\hline \multirow[t]{2}{*}{ U6 } & Forward & 5'- CTCGCTTCGGCAGCACA-3' \\
\hline & Reverse & 5'- AACGCTTCACGAATTTGCGT-3' \\
\hline
\end{tabular}

\section{Cell counting kit-8 (CCK-8) assay}

Cell viability was tested using the CCK-8 assay (Junxin, Suzhou, China). In brief, cells were plated in a 96-well cell culture plate at a concentration of $4 \times 10^{3}$ cells per well (3 replicates for each experimental condition). After different treatments, $10 \mu \mathrm{l}$ CCK- 8 reagent was added to the cells in each well and incubated for $2 \mathrm{~h}$ in a $5 \% \mathrm{CO}_{2}$ humidified incubator at $37^{\circ} \mathrm{C}$. The absorbance of the cells at $450 \mathrm{~nm}$ was detected using a microplate reader (Bio-Rad, Hercules, USA).

\section{5-ethynyl-2'-deoxyuridine (EdU) incorporation assay}

The cell proliferation potential was assessed using an EdU kit (Junxin, Suzhou, China). In brief, cells were plated in a 48-well cell culture plate at a concentration of $5 \times 10^{4}$ cells per well (3 replicates for each experimental condition). After different treatments, $200 \mu \mathrm{l} \mathrm{EdU} \mathrm{re-}$ agent was added to the cells in each well and incubated for $2 \mathrm{~h}$ in a $5 \% \mathrm{CO}_{2}$ humidified incubator at $37^{\circ} \mathrm{C}$. The EdU analysis was performed according to the manufacturer's instructions. The images were captured using a fluorescence microscope (Olympus, Tokyo, Japan).

\section{Construction of reporter vectors and luciferase reporter assay}

The fragments of MEG3 and PTEN containing the putative miR-93 binding site were amplified and inserted into the psiCheck2 Dual-Luciferase miRNA Target Expression Vector (Promega, Madison, WI, USA) to construct the reporter vectors MEG3-wild-type (MEG3-wt) and PTEN-wild-type (PTEN-wt), respectively. The psiCheck2 vectors cloned with the fragments of MEG3 and PTEN containing the mutant miR-93 binding site were referred to as MEG3-mutant-type (MEG3-mut) and PTENmutant-type (PTEN-mut), respectively. These reporters were co-transfected into HEK293 cells with miR-93 mimics using Lipofectamine 2000. The luciferase activities were measured with a Dual-Luciferase ${ }^{\bullet}$ Reporter Assay System Protocol (Promega).
RNA immunoprecipitation (RIP) assay

The RIP analysis was performed with the RIP kit (Millipore, MA USA) and anti-Argonaute 2 (Ago2) antibodies (Abcam, USA) according to the protocol. In brief, the cells were lysed in RNA lysis buffer, and the cell lysate was incubated with Protein A/G beads (Biolinkedin, Shanghai, China) and anti-Argonaute 2 (Ago2) antibodies (Abcam, USA) at $4{ }^{\circ} \mathrm{C}$ overnight. IgG was added as a negative control. The immunoprecipitated RNA was isolated and reverse transcribed, and qPCR analysis was introduced to measure the enrichment of MEG3 and miR-93.

\section{Enzyme-linked immunosorbent assay (ELISA)}

After different treatments, the culture supernatants were harvested, and the concentrations of the inflammatory cytokines of interleukin-6 (IL6), tumour necrosis factor alpha $(\mathrm{TNF} \alpha)$ and testosterone were tested using ELISA kits (Thermo Fisher Scientific, Waltham, USA) according to the manufacturer's protocol.

\section{Statistical analysis}

All experiments were repeated three times. Data are expressed as the mean \pm standard deviation (SD). Graphpad Prism 5 software (GraphPad, San Diego, CA) was used to perform the statistical analysis. The $p$-values of two independent comparisons were compared using Student's t-test, and multi-group comparisons were expressed using one-way ANOVA. A p-value of $<0.05$ indicated a significant difference.

\section{Results}

LPS induced dysfunction and inhibited cell growth in Leydig cells

To determine the role of LPS in Leydig cells, we treated Leydig cells with different concentrations of LPS $(0,50$, 100,200 , and $400 \mu \mathrm{g} / \mathrm{ml}$ ) for $48 \mathrm{~h}$. ELISA analysis demonstrated that LPS induced the secretion of TNF $\alpha$ and IL6 in Leydig cells (Fig. 1a and b). Further research 

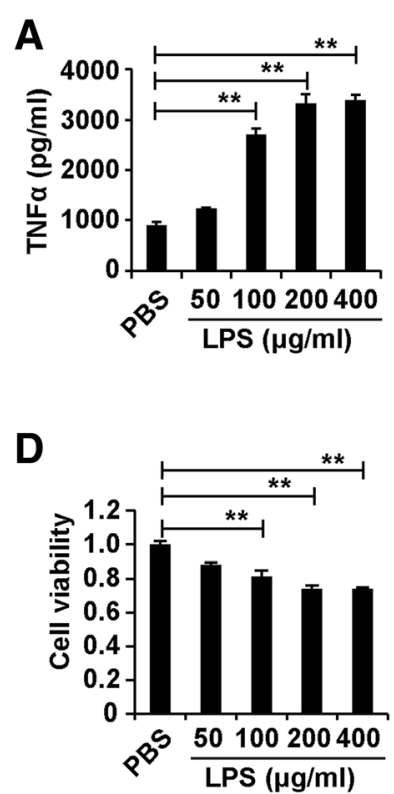
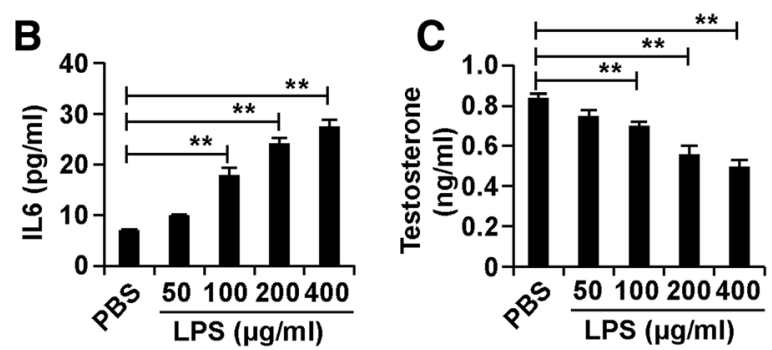

$\mathbf{E}$

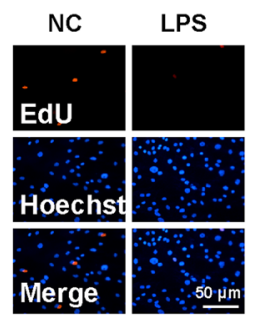

$\mathbf{F}$

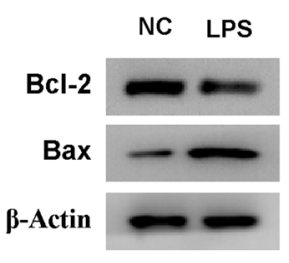

Fig. 1 LPS induced dysfunction and inhibited cell growth in Leydig cells. (A) ELISA analysis of secretion of TNFa and IL6 in Leydig cells. (B) ELISA analysis of testosterone production in Leydig cells. (C) CCK8 analysis of cell viability in Leydig cells. (D) EdU analysis of cell proliferation in Leydig cells. (E) Western blot analysis of cell apoptosis in Leydig cells. For A-C, Leydig cells were treated with different concentrations of LPS (0, 50, 100, $200,400 \mu \mathrm{g} / \mathrm{ml}$ ) for $48 \mathrm{~h}$. For D and E, Leydig cells were treated with LPS at a dose of $200 \mu \mathrm{g} / \mathrm{ml}$ for $48 \mathrm{~h}$. $\beta$-Actin was measured as a loading control for western blot analysis. "**" represents $p<0.01$, which indicates significantly statistic differences between two groups in Student's t-test

demonstrated that LPS inhibited the testosterone production in Leydig cells (Fig. 1c). The results of CCK8 analysis showed that LPS reduced the cell viability of Leydig cells (Fig. 1d). The results of EdU analysis showed that LPS decreased the cell proliferation of Leydig cells (Fig. 1e). Ultimately, western blot analysis revealed that LPS induced cell apoptosis, decreased Bcl-2 and increased Bax in Leydig cells (Fig. 1f). In summary, these results suggested that LPS induced dysfunction in Leydig cells.

\section{Silencing of MEG3 attenuated the role of LPS in Leydig cells}

To investigate whether MEG3 was involved in the role of LPS in Leydig cells, we measured the expression of MEG3 in Leydig cells treated with different concentrations of LPS. The results of qPCR analysis demonstrated that LPS increased the expression level of MEG3 in Leydig cells (Fig. 2a). This observation indicated that MEG3 participated in the role of LPS in Leydig cells.

To test whether MEG3 affected the role of LPS in Leydig cells, we first designed and synthesized short-hair RNA specific against MEG3 (shMEG3s) and control short-hair RNA (shNC). We transfected shMEGs and shNC into Leydig cells for $48 \mathrm{~h}$. The results of qPCR analysis demonstrated that both shMEG3s could efficiently downregulate the expression of MEG3 in Leydig cells, and shMEG3-1 was chosen for further experiments (Fig. 2b).

We divided the cells into four groups: shNC, shMEG3, shNC+LPS and shMEG3 + LPS. The ELISA analysis demonstrated that silencing of MEG3 decreased the secretion of TNF $\alpha$ and IL6 and inhibited LPS-induced promotion of TNF $\alpha$ and IL6 secretion in Leydig cells (Fig. 2c). Furthermore, knockdown of MEG3 elevated testosterone production and lowered LPS-induced testosterone production in Leydig cells (Fig. 2d). In addition, suppression of MEG3 enhanced the cell viability and proliferation and attenuated the LPS-induced inhibition of cell viability and proliferation in Leydig cells (Fig. 2e and $\mathrm{f}$ ).

\section{MEG3 absorbed miR-93-5p and miR-93-5p alleviated the role of LPS in Leydig cells}

To dissect the molecular mechanism of MEG3 in LPS in Leydig cells, we used the miRNAs prediction online site to screen the candidate targets of MEG3 and found that miR-93-5p was a candidate of MEG3. We introduced the luciferase reporter system to confirm the interaction between MEG3 and miR-93-5p. First, we cloned MEG3 transcripts containing the predicted binding site of miR93-5p and the mutant binding site of miR-93-5p into the luciferase reporter vector psiCheck2, referred to as psiCheck2-MEG3 wild type (psiCheck2-MEG3-wt) and psiCheck2-MEG3 mutant type (psiCheck2-MEG3-mut), 
A

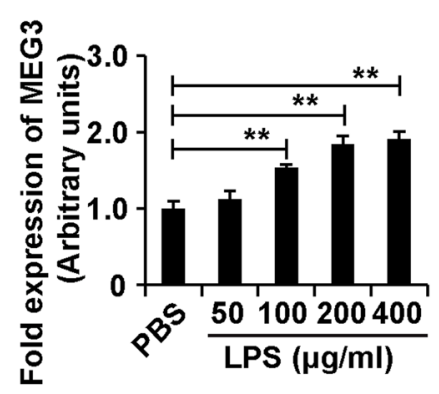

B

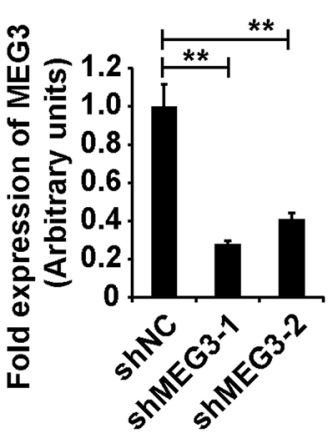

C

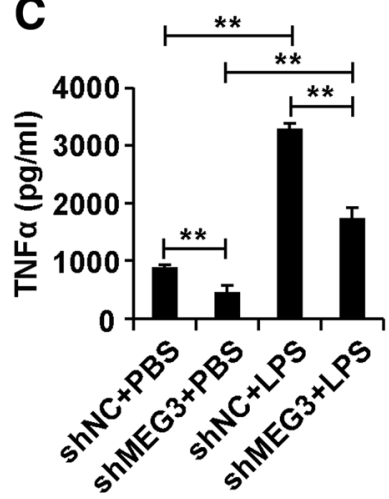

E

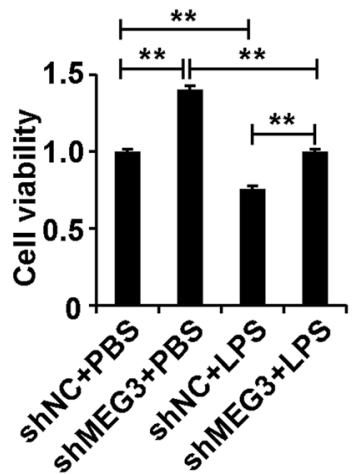

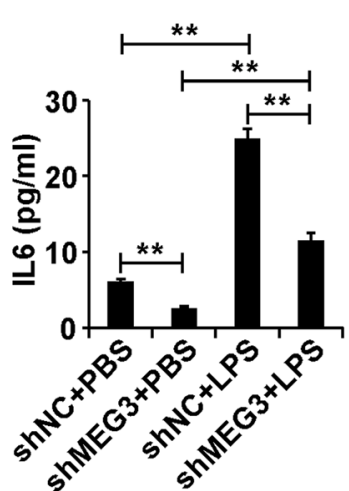

D

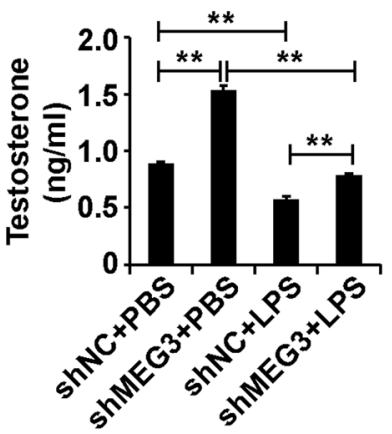

F
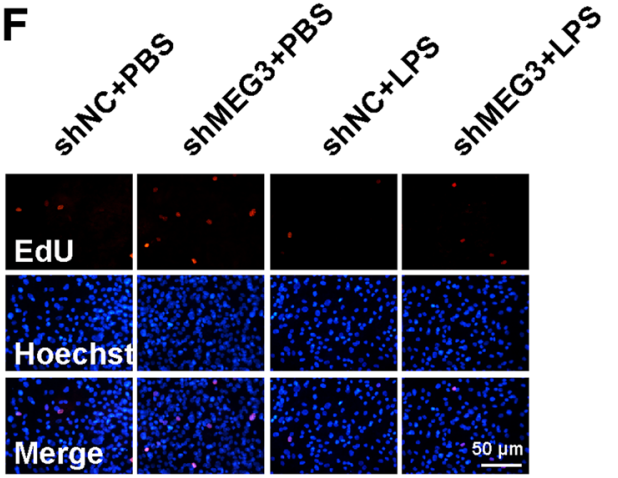

Fig. 2 Silencing of MEG3 attenuated the role of LPS in Leydig cells. (A) qPCR analysis of MEG3 expression in Leydig cells treated with different concentrations of LPS $(0,50,100,200,400 \mu \mathrm{g} / \mathrm{ml})$ for $48 \mathrm{~h}$. (B) qPCR analysis of MEG3 expression in Leydig cells transfected with siMEG3s and siNC for 48 h. (C) ELISA analysis of secretion of TNFa and IL6 in Leydig cells. (D) ELISA analysis of testosterone production in Leydig cells. (E) CCK8 analysis of cell viability in Leydig cells. (F) EdU analysis of cell proliferation in Leydig cells. For C-F, Leydig cells were divided into four groups: shNC, shMEG3, shNC+LPS and shMEG3 + LPS. $\beta$-Actin was measured as an internal control for qPCR analysis. "*** represents $p<0.01$, which indicates statistically significant differences between two groups in Student's t-test

respectively. We transfected psiCheck2-MEG3-wt and psiCheck2-MEG3-mut together with miR-93-5p mimics into 293 cells. At $48 \mathrm{~h}$, the luciferase activity was measured, and the results demonstrated that miR-93-5p mimics inhibited the luciferase activity of psiCheck2MEG3-wt, and the luciferase activity was restored in psiCheck2-MEG3-mut (Fig. 3a). Taken together, these results demonstrated that miR-93-5p could bind to the predicted binding site of miR-93-5p on MEG3 transcripts.

To investigate whether miR-93-5p affected the role of LPS on Leydig cells, we first measured the expression of miR-93-5p in Leydig cells treated with different concentrations of LPS, and the results of qPCR analysis demonstrated that LPS decreased the expression level of miR-93-5p in Leydig cells (Fig. 3b). This observation indicated that miR- 


\section{A}

MEG3-wt: 3'...TATCTTTCACGAGACCTTT...5'

- IIIIIIII

: I: I: I:

MEG3-mut:3'...TATCATACUCCAGACCTTT...5'

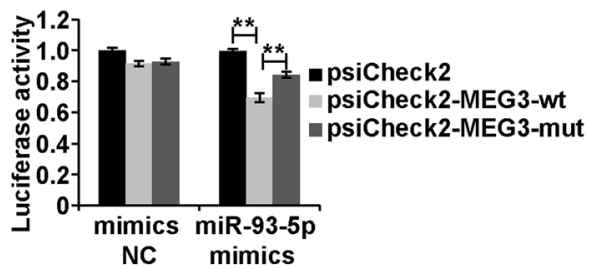

C

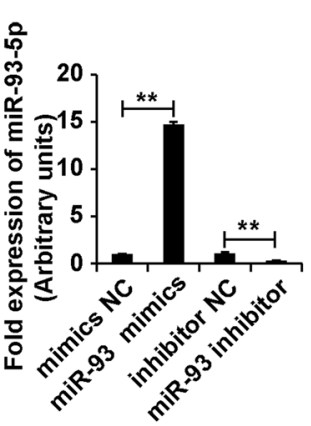

B

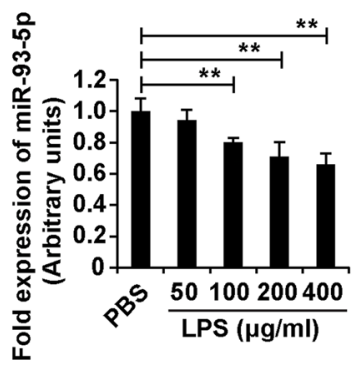

E

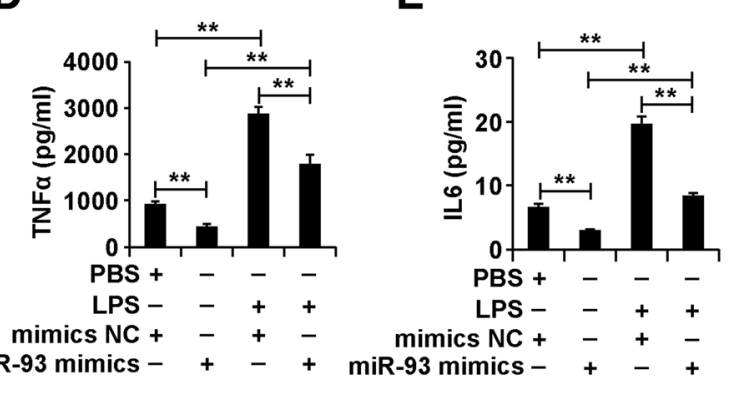

F
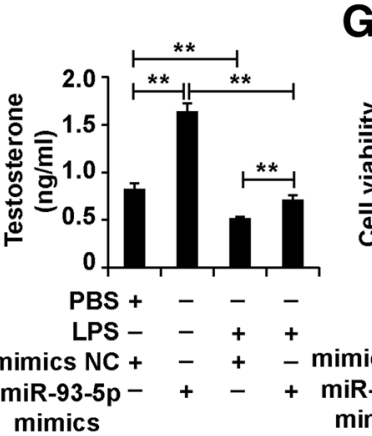

G

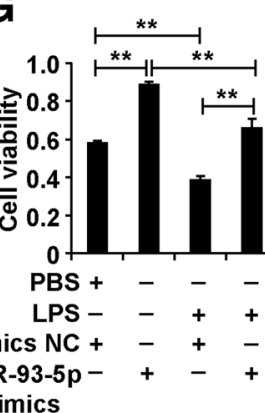

H

PBS +

PBS +
LPS $\operatorname{mimics} N C+$
$\operatorname{miR}-93-5 p-$ mimics

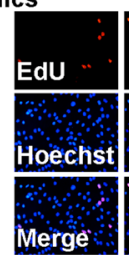

Fig. 3 MEG3 absorbed miR-93-5p and miR-93-5p alleviated the role of LPS in Leydig cells. (A and B) Luciferase reporter system analysis of the interaction between MEG3 and miR-93-5p. (C) qPCR analysis of miR-93-5p expression in Leydig cells treated with different concentrations of LPS $(0,50,100,200,400 \mu \mathrm{g} / \mathrm{ml})$ for $48 \mathrm{~h}$. (D) qPCR analysis of miR-93-5p expression in Leydig cells transfected with miR-93-5p mimics, mimics NC, miR93-5p inhibitor and inhibitor NC for 48 h. (E) ELISA analysis of secretion of TNFa and IL6 in Leydig cells. (F) ELISA analysis of testosterone production in Leydig cells. (G) CCK8 analysis of cell viability in Leydig cells. (H) EdU analysis of cell proliferation in Leydig cells. For E-H, Leydig cells were divided into four groups: mimics NC, miR-93-5p mimics, mimics NC + LPS and miR-93-5p mimics+LPS. U6 was measured as an internal control for qPCR analysis. "*** represents $p<0.01$, which indicates statistically significant differences between two groups in Student's t-test

93-5p participated in the role LPS in Leydig cells. We synthesized the miR-93-5p mimics, mimics NC, miR-93-5p inhibitor and inhibitor NC, and the results of qPCR analysis demonstrated that the miR-93-5p mimics significantly increased the miR-93-5p level and the miR-93-5p inhibitor significantly decreased that in Leydig cells (Fig. 3c).

In addition, we divided the cells into four groups: mimics NC, miR-93-5p mimics, mimics NC+LPS and miR-93-5p mimics+LPS. The ELISA analysis demonstrated that miR-93-5p decreased the secretion of TNF $\alpha$ and IL6 and inhibited the LPS- induced promotion of TNF $\alpha$ and IL6 secretion in Leydig cells (Fig. 3d and e). Furthermore, miR-93-5p increased the testosterone production and decreased the LPS-induced testosterone production of Leydig cells (Fig. 3f). In addition, miR-93-5p enhanced the cell viability and proliferation and attenuated the LPS-induced inhibition of cell viability and proliferation in Leydig cells (Fig. $3 g$ and h). Taken together, these results showed that MEG3 absorbed miR-93$5 p$ and that miR-93-5p alleviated the role of LPS on Leydig cells. 
Inhibition of miR-93-5p alleviated the role of silenced MEG3 in Leydig cells treated with LPS

To investigate whether miR-93-5p affected the role of MEG3 in Leydig cells treated with LPS, we divided the cells into six groups: shNC+inhibitor NC, shMEG3 + inhibitor NC, shMEG3+ miR-93-5p inhibitor, shNC+inhibitor NC + LPS, shMEG3 + inhibitor NC + LPS, and shMEG3+ miR-93-5p inhibitor+LPS. The ELISA analysis demonstrated that miR-93-5p inhibitor restored the shMEG3-induced inhibition of TNF $\alpha$ and IL6 secretion of Leydig cells (Fig. 4a and b). Furthermore, the ELISA analysis demonstrated that miR-93-5p inhibitor alleviated the shMEG3-induced elevation of testosterone production of Leydig cells (Fig. 4c). In addition, miR-93-5p inhibitor attenuated the shMEG3-induced enhancement of cell viability and proliferation in Leydig cells (Fig. $4 \mathrm{~d}$
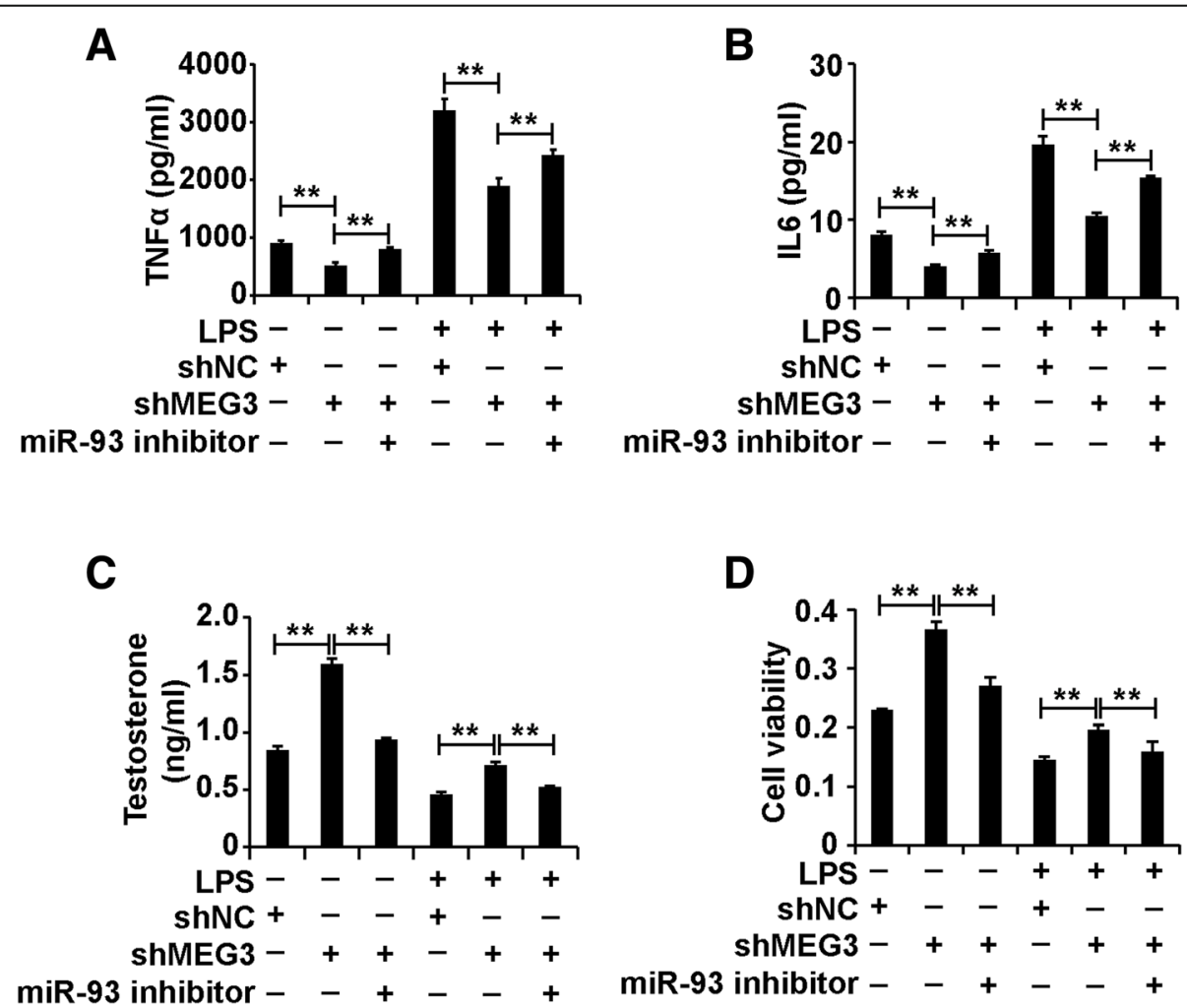

$\mathbf{D}$

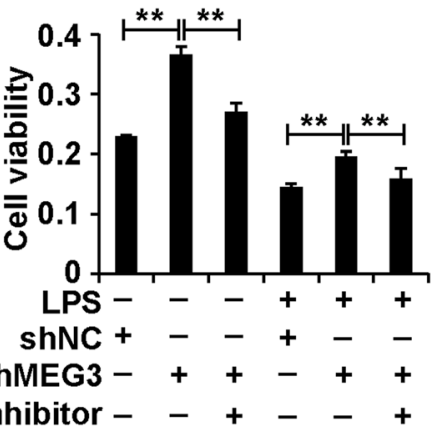

E

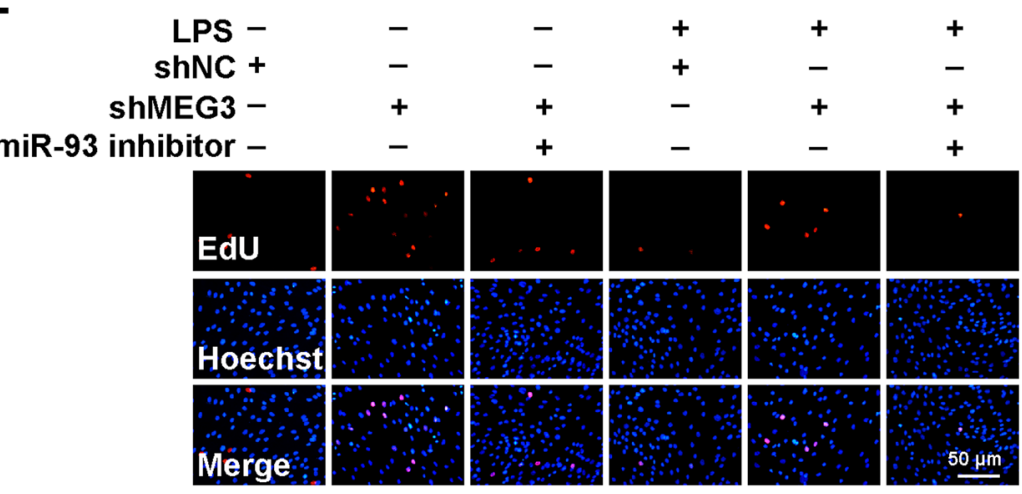

Fig. 4 Inhibition of miR-93-5p alleviated the role of silenced MEG3 in Leydig cells treated with LPS. (A) ELISA analysis of secretion of TNFa and IL6 in Leydig cells. (B) ELISA analysis of testosterone production in Leydig cells. (C) CCK8 analysis of cell viability in Leydig cells. (D) EdU analysis of cell proliferation in Leydig cells. For A-D, Leydig cells were divided into six groups: shNC+inhibitor NC, shMEG3 + inhibitor NC, shMEG3+ miR-93$5 p$ inhibitor, shNC+inhibitor NC+ LPS, shMEG3 + inhibitor NC+ LPS, and shMEG3+ miR-93-5p inhibitor+LPS. "***" represents $p<0.01$, which indicates statistically significant differences between two groups in Student's t-test 
and e). In summary, these results suggested that inhibition of miR-93-5p alleviated the role of silenced MEG3 in Leydig cells treated with LPS.

\section{miR-93-5p regulated PTEN expression in Leydig cells}

To investigate the molecular mechanism of miR-93-5p in Leydig cells, we used the miRNAs prediction online site to screen the candidate target of miR-93-5p and found that PTEN was a candidate of miR-93-5p. To detect whether miR-93-5p could regulate the PTEN expression, we transfected the miR-93-5p mimics, mimics NC, miR-93-5p inhibitor and inhibitor NC into Leydig cells. The results of qPCR and western blots analysis showed that the miR-93-5p mimics inhibited and miR93-5p inhibitor promoted the PTEN expression in Leydig cells (Fig. $5 \mathrm{a}$ and $\mathrm{b}$ ). To further confirm the interaction between miR-93-5p and PTEN, we applied the luciferase reporter system as a test. First, we cloned the PTEN 3' UTR containing the predicted binding site of miR-93-5p and the mutant binding site of miR-93-5p into the luciferase reporter vector psiCheck2, known as psiCheck2-PTEN wild type (psiCheck2-PTEN-wt) and psiCheck2-PTEN mutant type (psiCheck2-PTEN-mut), respectively. We transfected psiCheck2-PTEN-wt and psiCheck2-PTEN-mut together with miR-93-5p mimics into 293 cells. At $48 \mathrm{~h}$, the luciferase activity was measured. The results demonstrated that miR-93-5p mimics inhibited the luciferase activity of psiCheck2PTEN-wt, and the luciferase activity was restored in psiCheck2-PTEN-mut (Fig. 5c and d). Taken together, these results demonstrated that miR-93-5p inhibited PTEN expression in Leydig cells.

Over-expression of PTEN attenuated the role of miR-93$5 p$ mimics in Leydig cells treated with LPS

To investigate whether PTEN affected the role of miR93-5p in Leydig cells treated with LPS, we first detected the PTEN expression in Leydig cells treated with different concentrations of LPS. The results of $\mathrm{qPCR}$ and western blot analyses showed that LPS elevated the expression level of PTEN in Leydig cells (Fig. 6a), indicating that PTEN was involved in the role of LPS in Leydig cells.

Furthermore, we constructed overexpression clones of PTEN based on the plvx vector (plvx-PTEN), and qPCR and western blot analyses revealed that overexpression clones of PTEN significantly elevated the expression level of PTEN in Leydig cells (Fig. 6b).

We divided the cells into four groups: mimics NC, LPS + mimics NC, LPS + miR-93-5p mimics and LPS + miR-93-5p mimics+plvx-PTEN. The ELISA analysis demonstrated that plvx-PTEN restored miR-93-5p
A

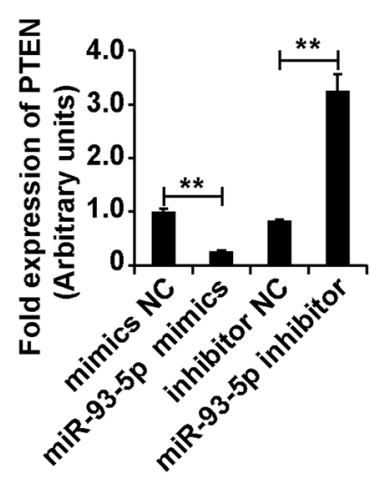

C

PTEN-wt: 5'...GGAUUAAUAAAGAUGGCACUUUC...3'
miR-93-5p : 3' GAUGGACGUGCUUGUCGUGAAAC 5'
PTEN-mut: 5'...GGAUUAAUAAAGAUGCCUCAUAC...3'

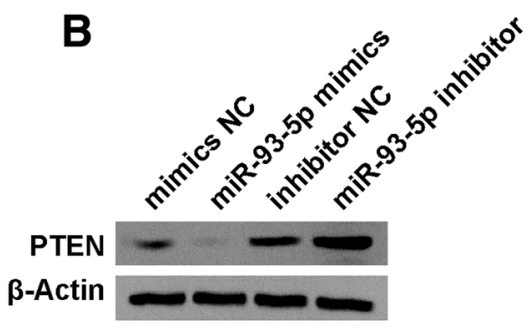

D $\quad$ psiCheck2

$\square$ psiCheck2-PTEN-wt

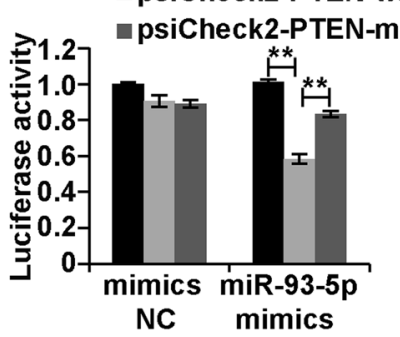

Fig. 5 miR-93-5p regulated PTEN expression in Leydig cells. (A and B) qPCR and western blot analysis of PTEN expression in Leydig cells transfected with miR-93-5p mimics, mimics NC, miR-93-5p inhibitor and inhibitor NC for $48 \mathrm{~h}$. (C and D) Luciferase reporter system analysis of the interaction between MEG3 and miR-93-5p. $\beta$-Actin was measured as a loading control for western blot analysis. "***" represents $p<0.01$, which indicates statistically significant differences between two groups in Student's t-test 

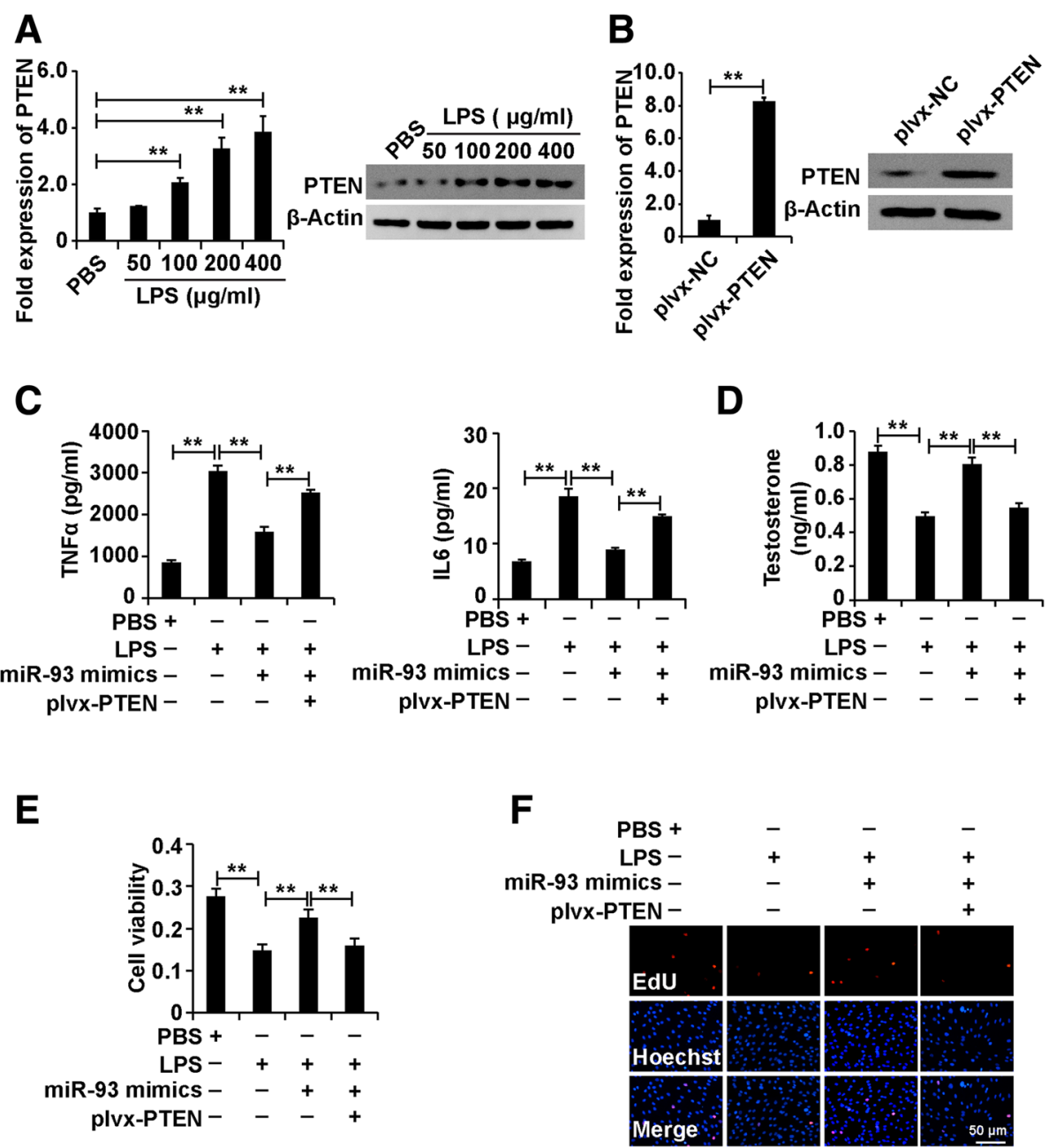

Fig. 6 Over-expression of PTEN attenuated the role of miR-93-5p mimics in Leydig cells treated with LPS. (A) qPCR and western blot analysis of PTEN expression in Leydig cells treated with different concentrations of LPS $(0,50,100,200,400 \mu \mathrm{g} / \mathrm{ml})$ for $48 \mathrm{~h}$. (B) qPCR and western blot analysis of PTEN expression in Leydig cells transfected with plvx-PTEN and plvx-NC for $48 \mathrm{~h}$. (C) ELISA analysis of secretion of TNFa and IL6 in Leydig cells. (D) ELISA analysis of testosterone production in Leydig cells. (E) CCK8 analysis of cell viability in Leydig cells. (F) EdU analysis of cell proliferation in Leydig cells. For C-F, Leydig cells were divided into four groups: mimics NC, LPS + mimics NC, LPS+ miR-93-5p mimics and LPS + miR-93-5p mimics+plvx-PTEN. $\beta$-Actin was measured as an internal control for qPCR analysis. "**" represents $p<0.01$, which indicates statistically significant differences between two groups in Student's t-test

mimics-induced inhibition of TNFa and IL6 secretion of Leydig cells (Fig. 6c). Furthermore, the ELISA analysis demonstrated that plvx-PTEN alleviated miR-93-5p mimics -induced elevation of testosterone production in Leydig cells (Fig. 6d). In addition, plvx-PTEN attenuated miR-93-5p mimic-induced enhancement of cell viability and proliferation in Leydig cells (Fig. 6e and f). In summary, these observations revealed that upregulation of PTEN restored the role of miR-93-5p in Leydig cells treated with LPS.

\section{LPS activated the MEG3/miR-93-5p/PTEN signalling pathway in Leydig cells}

To determine whether MEG3 exerted its effect by regulating the miR-93-5p/PTEN pathway in Leydig cells treated with LPS, we first measured whether PTEN was regulated by MEG3/miR-93-5p pathway in Leydig cells treated with LPS. The results demonstrated that inhibition of LPS-induced PTEN by miR93-5 $p$ mimics was restored by overexpressed MEG3 (Fig. 7a). These results demonstrated that MEG3 could alleviate the repression of PTEN by miR-93-5p in Leydig cells treated with LPS.

We designed and synthesized short-hair RNA specific against PTEN (shPTENs) and control short-hair RNA (shNC). We transfected shPTENs and shNC into the Leydig cells for $48 \mathrm{~h}$. The results of qPCR analysis demonstrated that all three shPTENs could efficiently inhibit PTEN expression in Leydig cells, and shPTEN-3 was chosen for further experiments (Fig. 7b). 

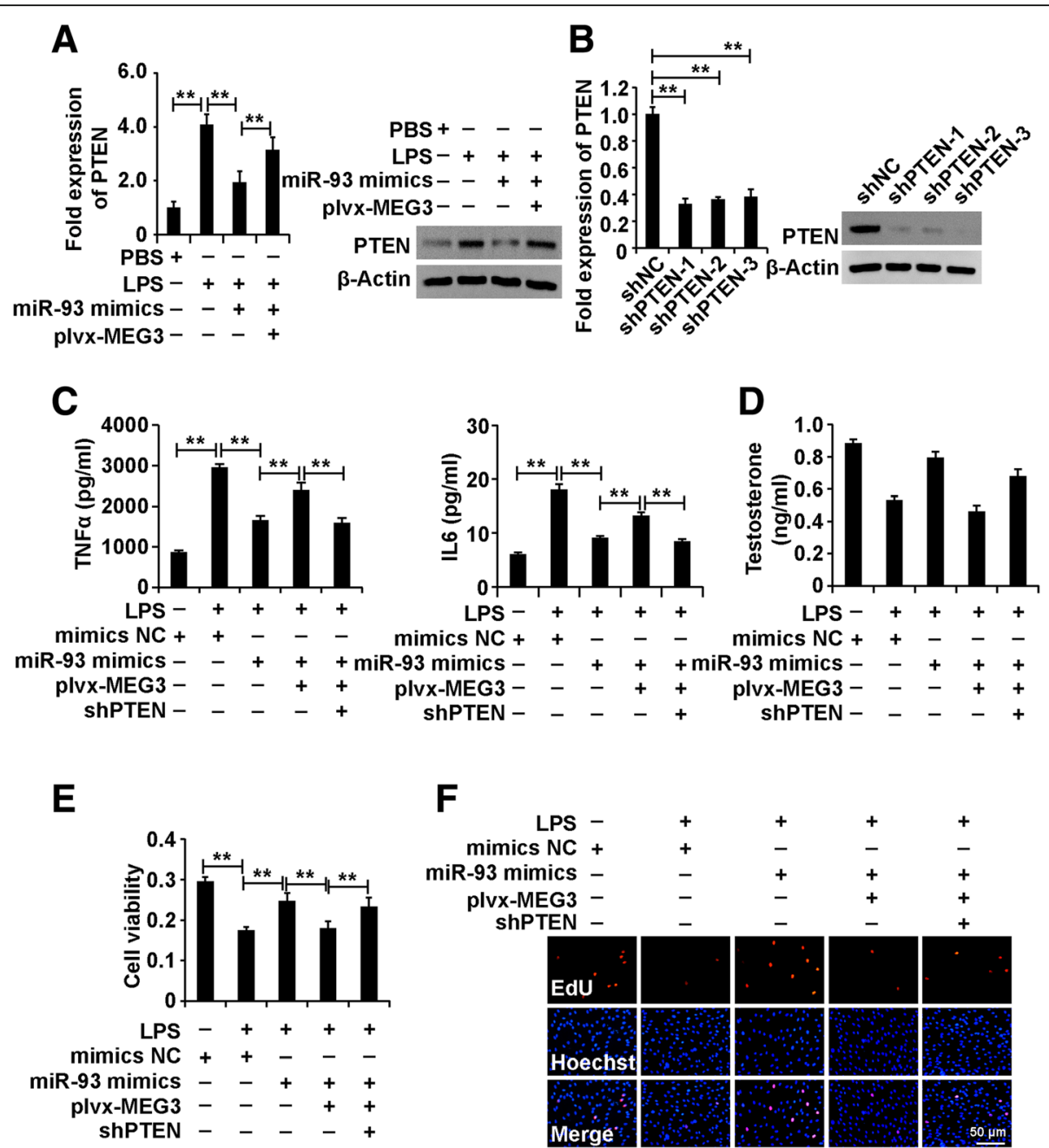

Fig. 7 LPS activates the MEG3/miR-93-5p/PTEN signalling pathway in Leydig cells. (A) qPCR and western blot analysis of PTEN expression in Leydig cells divided into four groups: mimics NC, LPS + mimics NC, LPS + miR-93-5p mimics and LPS + miR-93-5p mimics+plvx-MEG3. (B) qPCR and western blot analysis of PTEN expression in Leydig cells transfected with shPTENs and shNC for $48 \mathrm{~h}$. (C) ELISA analysis of secretion of TNFa and IL6 in Leydig cells. (D) ELISA analysis of testosterone production in Leydig cells. (E) CCK8 analysis of cell viability in Leydig cells. (F) EdU analysis of cell proliferation in Leydig cells. For C-F, Leydig cells were divided into five groups: mimics NC, LPS + mimics NC, LPS+ miR-93-5p mimics, LPS + miR-93-5p mimics+plvx-MEG3 and LPS + miR-93-5p mimics+plvx-MEG3 + shPTEN. $\beta$-Actin was measured as an internal control for qPCR analysis. "**" represents $p<0.01$, which indicates statistically significant differences between two groups in Student's t-test

To determine the role of the MEG3/miR-93-5p/ PTEN signalling pathway in Leydig cells treated with LPS, we divided the cells into five groups: mimics NC, LPS + mimics NC, LPS+ miR-93-5p mimics, LPS + miR-93-5p mimics+plvx-MEG3 and LPS + miR-93-5p mimics+plvx-MEG3 + shPTEN. The ELISA, CCK8 and EdU analyses demonstrated that any disturbers of the MEG3/miR-93-5p/PTEN signalling pathway affected the role of LPS in Leydig cells (Fig. 7c-f). In summary, these resulted revealed that LPS played a role via activation of the MEG3/miR-93-5p/PTEN signalling pathway in Leydig cells.

\section{Discussion}

Infertility is a heavy global public health threat that is estimated to affect one out of every twenty men [20]. The male factor, which contains exposure to hazardous chemicals and radiation, excessive alcohol consumption, tobacco smoking and other unhealthy lifestyle factors, accounts for more than $50 \%$ infertility due to decreased sperm quality [21]. In the testes, autoimmune orchitis, which is induced by systemic inflammation such as immunosuppression, inflammatory reactions and infection, leads to testicular dysfunction, germ cell apoptosis, and inhibition of Leydig cell steroidogenesis and spermatogenesis, thus resulting in male infertility $[2,22]$. 
Therefore, immune homeostasis is essential for normal function of the testes. This study demonstrated that LPS induced an increase of TNF $\alpha$ and IL6 secretion and a decrease of testosterone secretion, inhibited cell growth and induced apoptosis in Leydig cells. Therefore, an urgent need exists to investigate the role and underlying mechanism of LPS in the cellular progression and function of Leydig cells.

LncRNAs are divided into three main categories: intronic lncRNA (mainly produced in the intron region of the coding gene) intergenic lncRNA (mainly produced in the middle region of the two coding genes) and antisense lncRNA (mainly produced in the antisense strand of the coding gene) $[8,23]$. Recently, more numerous functional lncRNAs were identified in human diseases $[14,24]$. Several studies revealed the lncRNAs involved in spermatogenesis, steroidogenesis and cell differentiation in the testes. One study measured the RNA profile of human testicular cells to identify the lncRNAs associated with spermatogenesis [25]. Another research study systematically screened the enrichment of long noncoding RNAs that affected the differentiation of Sertoli and Leydig cells via transcriptome analysis of adults with Klinefelter syndrome (KS; 47,XXY) [17]. Yui Satoh and colleagues revealed that Tesra, which is a novel testisspecific lncRNA, regulated the Prss42/Tessp 2 gene and thus affected mouse spermatogenesis [26]. This study demonstrated that MEG3, a lncRNA, was increased in a LPS-treated mice model and in Leydig cells. Furthermore, knockdown of MEG3 alleviated the role of LPS in inflammatory factors and androgen secretion and cell growth of Leydig cells.

Recently, several regulatory mechanisms of lncRNAs were identified: combined with chromatin, which affects chromatin remodelling and generally affectis gene transcription; combined with the 5'UTR of genes, which affects gene transcription initiation; combined with mRNA transcripts, which affects mRNA alternative splicing, transport, and translation efficiency; binding to protein, which affects the location, modification and activity of protein; as a precursor of miRNA and siRNA, which affects the amount and activity of miRNA; and as a molecular sponge binding miRNA, which affects the amount of free miRNA, thus affecting the expression level of miRNA downstream target genes and activity [10, 27, 28].

The competing endogenous RNAs (ceRNA) hypothesis has attracted increasing attention from scientists [29]. The ceRNA hypothesis defined as that, IncRNAs which behaves binding site of miRNA serves as a molecular sponge to absorb the miRNA and thus, lowers the free miRNAs levels in cytoplasm, resulting in the decrease of the binding between miRNAs and its target-mRNA, leading to the elevation of gene transcripts and proteins
$[29,30]$. MEG3 was reported to exert its function via the ceRNA hypothesis [31]. Ru Yang and colleagues demonstrated that silencing of MEG3 alleviated LPS-induced apoptosis by sponging miR-21 in renal tubular epithelial cells [32]. This study demonstrated that MEG3 affected the role of LPS by sponging miR-93-5p, thus modulating PTEN expression in human Leydig cells.

PTEN (phosphatase and tensin homologue deleted on chromosome ten) was first identified as a tumour suppressor gene and has an anti-growth function in a variety of cellular processes [33-35]. PTEN modulates the PI3K/AKT signalling pathway [36, 37]. Pingping Xue and colleagues demonstrated that PTEN which was induced by LPS inhibited the trophoblast invasion via AP$1 / \mathrm{NF}-\mathrm{kB}$ pathway inhibits trophoblast invasion [38]. However, the regulation of PTEN remains largely unclear. This study demonstrated that PTEN is regulated by LPS-induced MEG3, which absorbs miR-93-5p, thus supplying a novel regulatory mechanism of PTEN.

\section{Conclusions}

This study first introduced LPS for treatment of Leydig cells to simulate Leydig cells in the orchitis environment. Additionally, this study demonstrated that MEG3 expression was increased in Leydig cells under LPS and that silencing of MEG3 attenuated the role of LPS in Leydig cells. Finally, this study revealed that MEG3 exerted its function via modulation of the miR-93-5p/ PTEN pathway in Leydig cells under LPS, thus indicating the potential of this signalling pathway in treatment of orchitis.

\section{Abbreviations}

MEG3: Maternally expressed gene 3; LPS: Lipopolysaccharides; NcRNAs: Noncoding RNAs; LncRNAs: Long ncRNAs; DMEM: Dulbecco's modified Eagle

medium; FBS: Foetal bovine serum; siRNAs: Small interfering RNA; RIPA: Radio immunoprecipitation assay; SDS-PAGE: Sodium dodecyl sulfate-

polyacrylamide gel electrophoresis; PVDF: Polyvinylidene fluoride; ECL: Chemiluminescence; CCK-8: Cell counting kit-8; EdU: 5-ethynyl-2'deoxyuridine; RIP: RNA immunoprecipitation; ELISA: Enzyme-linked immunosorbent assay

\section{Acknowledgements}

All people contributed towards the article were listed in the authorship.

\section{Authors' contributions}

Xu Zhou, Xiongbing Zu and Nenghui Liu designed research; Xu Zhou, Jingliang He, Jinbo Chen performed experiments; Xu Zhou, Jingliang He and Zhenyu Ou analyzed data, interpreted results and figures; Xu Zhou drafted manuscript; Xu Zhou, Zhenyu Ou, Yu Cui, Xiongbing Zu and Nenghui Liu approved final version of manuscript.

\section{Funding}

This work was granted by National Nature Science foundation of the People's Republic of China (No. 81700665, 81873626 and 81902592).

\section{Availability of data and materials}

The data underlying this article will be shared on reasonable request to the corresponding author. 


\section{Ethics approval and consent to participate}

The research was approved and supervised by the Ethical Committee of Xiangya Hospital, Central South University.

\section{Consent for publication}

All authors are honored to publish this article entitled "Silencing of MEG3 attenuated the role of lipopolysaccharides by modulating the miR-93-5p/ PTEN pathway in Leydig cells"in the "Reproductive Biology and Endocrinology".

\section{Competing interests}

The authors declare that there is no conflict of interests.

Received: 16 September 2020 Accepted: 11 February 2021 Published online: 27 February 2021

\section{References}

1. Trojian TH, Lishnak TS, Heiman D. Epididymitis and orchitis: an overview. Am Fam Physician. 2009;79:583-7.

2. Schuppe HC, Meinhardt A, Allam JP, Bergmann M, Weidner W, Haidl G. Chronic orchitis: a neglected cause of male infertility? Andrologia. 2008;40: 84-91.

3. Zirkin BR, Papadopoulos V. Leydig cells: formation, function, and regulation. Biol Reprod. 2018;99:101-11.

4. Bertani B, Ruiz N. Function and biogenesis of lipopolysaccharides. EcoSal Plus. 2018;8

5. Reddy MM, Mahipal SV, Subhashini J, Reddy MC, Roy KR, Reddy GV, Reddy PR, Reddanna P. Bacterial lipopolysaccharide-induced oxidative stress in the impairment of steroidogenesis and spermatogenesis in rats. Reprod Toxicol. 2006:22:493-500

6. O'Bryan MK, Schlatt S, Phillips DJ, de Kretser DM, Hedger MP. Bacterial lipopolysaccharide-induced inflammation compromises testicular function at multiple levels in vivo. Endocrinology. 2000;141:238-46

7. Mattick JS, Makunin IV: Non-coding RNA. Hum Mol Genet 2006, 15 Spec No $1: R 17-29$.

8. Mattick JS, Rinn JL. Discovery and annotation of long noncoding RNAs. Nat Struct Mol Biol. 2015;22:5-7.

9. Weinberg Z, Ruzzo WL. Sequence-based heuristics for faster annotation of non-coding RNA families. Bioinformatics. 2006;22:35-9.

10. Guttman M, Rinn JL. Modular regulatory principles of large non-coding RNAs. Nature. 2012;482:339-46.

11. Han P, Chang CP. Long non-coding RNA and chromatin remodeling. RNA Biol. 2015:12:1094-8.

12. Mercer TR, Dinger ME, Mattick JS. Long non-coding RNAs: insights into functions. Nat Rev Genet. 2009:10:155-9.

13. Quinn JJ, Chang HY. Unique features of long non-coding RNA biogenesis and function. Nat Rev Genet. 2016:17:47-62.

14. Shi $X$, Sun M, Liu H, Yao Y, Song Y. Long non-coding RNAs: a new frontier in the study of human diseases. Cancer Lett. 2013;339:159-66.

15. Rogoyski OM, Pueyo Jl, Couso JP, Newbury SF. Functions of long noncoding RNAs in human disease and their conservation in Drosophila development. Biochem Soc Trans. 2017:45:895-904.

16. Esteller M. Non-coding RNAs in human disease. Nat Rev Genet. 2011;12: 861-74

17. Winge SB, Dalgaard MD, Belling KG, Jensen JM, Nielsen JE, Aksglaede L, Schierup MH, Brunak S, Skakkebaek NE, Juul A, et al. Transcriptome analysis of the adult human Klinefelter testis and cellularity-matched controls reveals disturbed differentiation of Sertoli- and Leydig cells. Cell Death Dis. 2018:9: 586.

18. Benetatos L, Vartholomatos G, Hatzimichael E. MEG3 imprinted gene contribution in tumorigenesis. Int J Cancer. 2011;129:773-9.

19. Wang Z, Chi X, Liu L, Wang Y, Mei X, Yang Y, Jia T. Long noncoding RNA maternally expressed gene 3 knockdown alleviates lipopolysaccharideinduced inflammatory injury by up-regulation of miR-203 in ATDC5 cells. Biomed Pharmacother. 2018:100:240-9.

20. Ombelet W, Cooke I, Dyer S, Serour G, Devroey P. Infertility and the provision of infertility medical services in developing countries. Hum Reprod Update. 2008;14:605-21.

21. Merzenich $\mathrm{H}$, Zeeb $\mathrm{H}$, Blettner M. Decreasing sperm quality: a global problem? BMC Public Health. 2010;10:24.
22. Pentikainen V, Erkkila K, Suomalainen L, Otala M, Pentikainen MO, Parvinen M, Dunkel L. TNFalpha down-regulates the Fas ligand and inhibits germ cell apoptosis in the human testis. J Clin Endocrinol Metab. 2001;86:4480-8.

23. Sun M, Gadad SS, Kim DS, Kraus WL. Discovery, annotation, and functional analysis of long noncoding RNAs controlling cell-cycle gene expression and proliferation in breast Cancer cells. Mol Cell. 2015;59:698-711.

24. Liao Y, Shen L, Zhao H, Liu Q, Fu J, Guo Y, Peng R, Cheng L. LncRNA CASC2 interacts with miR-181a to modulate Glioma growth and resistance to TMZ through PTEN pathway. J Cell Biochem. 2017;118:1889-99.

25. Rolland AD, Evrard B, Darde TA, Le Beguec C, Le Bras Y, Bensalah K, Lavoue S, Jost B, Primig M, Dejucq-Rainsford N, et al. RNA profiling of human testicular cells identifies syntenic IncRNAs associated with spermatogenesis. Hum Reprod. 2019:34:1278-90.

26. Satoh Y, Takei N, Kawamura S, Takahashi N, Kotani T, Kimura AP. A novel testis-specific long noncoding RNA, Tesra, activates the Prss42/Tessp-2 gene during mouse spermatogenesisdagger. Biol Reprod. 2019;100:833-48.

27. Bunch $\mathrm{H}$. Gene regulation of mammalian long non-coding RNA. Mol Gen Genomics. 2017.

28. Wilusz JE, Sunwoo H, Spector DL. Long noncoding RNAs: functional surprises from the RNA world. Genes Dev. 2009:23:1494-504.

29. Salmena L, Poliseno L, Tay Y, Kats L, Pandolfi PP. A ceRNA hypothesis: the Rosetta stone of a hidden RNA language? Cell. 2011;146:353-8.

30. Tay Y, Rinn J, Pandolfi PP. The multilayered complexity of ceRNA crosstalk and competition. Nature. 2014:505:344-52.

31. Huang P, Huang FZ, Liu HZ, Zhang TY, Yang MS, Sun CZ. LncRNA MEG3 functions as a ceRNA in regulating hepatic lipogenesis by competitively binding to miR-21 with LRP6. Metabolism. 2019;94:1-8.

32. Yang R, Liu S, Wen J, Xue L, Zhang Y, Yan D, Wang G, Liu Z. Inhibition of maternally expressed gene 3 attenuated lipopolysaccharide-induced apoptosis through sponging miR-21 in renal tubular epithelial cells. J Cell Biochem. 2018:119:7800-6.

33. Steck PA, Pershouse MA, Jasser SA, Yung WK, Lin H, Ligon AH, Langford LA, Baumgard ML, Hattier T, Davis $T$, et al. Identification of a candidate tumour suppressor gene, MMAC1, at chromosome 10q23.3 that is mutated in multiple advanced cancers. Nat Genet. 1997:15:356-62.

34. Li J, Hu K, Gong G, Zhu D, Wang Y, Liu H, Wu X. Upregulation of MiR-205 transcriptionally suppresses SMAD4 and PTEN and contributes to human ovarian cancer progression. Sci Rep. 2017;7:41330.

35. Jiang XM, Yu XN, Liu TT, Zhu HR, Shi X, Bilegsaikhan E, Guo HY, Song GQ, Weng SQ. Huang XX, et al: microRNA-19a-3p promotes tumor metastasis and chemoresistance through the PTEN/Akt pathway in hepatocellular carcinoma. Biomed Pharmacother. 2018:105:1147-54

36. Song MS, Salmena L, Pandolfi PP. The functions and regulation of the PTEN tumour suppressor. Nat Rev Mol Cell Biol. 2012;13:283-96.

37. Liu H, Wang J, Tao Y, Li X, Qin J, Bai Z, Chi B, Yan W, Chen X. Curcumol inhibits colorectal cancer proliferation by targeting miR-21 and modulated PTEN/PI3K/Akt pathways. Life Sci. 2019;221:354-61.

38. Xue P, Fan W, Diao Z, Li Y, Kong C, Dai X, Peng Y, Chen L, Wang H, Hu Y, $\mathrm{Hu}$ Z. Up-regulation of PTEN via LPS/AP-1/NF-kappaB pathway inhibits trophoblast invasion contributing to preeclampsia. Mol Immunol. 2020;118: 182-90.

\section{Publisher's Note}

Springer Nature remains neutral with regard to jurisdictional claims in published maps and institutional affiliations.

Ready to submit your research? Choose BMC and benefit from

- fast, convenient online submission

- thorough peer review by experienced researchers in your field

- rapid publication on acceptance

- support for research data, including large and complex data types

- gold Open Access which fosters wider collaboration and increased citations

- maximum visibility for your research: over $100 \mathrm{M}$ website views per year

At $\mathrm{BMC}$, research is always in progress.

Learn more biomedcentral.com/submissions 